\title{
A FINITE DIFFERENCE SCHEME FOR A FLUID DYNAMIC TRAFFIC FLOW MODEL APPENDED WITH TWO-POINT BOUNDARY CONDITION
}

\author{
M. O. Gani, M. M. Hossain and L. S. Andallah \\ Department of Mathematics \\ Jahangirnagar University, Savar, Dhaka, Bangladesh \\ e-mail: andallahls@gmail.com,osmangani2@yahoo.com
}

Received 19.10.2010

Accepted 16.07.2011

\begin{abstract}
A fluid dynamic traffic flow model with a linear velocity-density closure relation is considered. The model reads as a quasi-linear first order hyperbolic partial differential equation (PDE) and in order to incorporate initial and boundary data the PDE is treated as an initial boundary value problem (IBVP). The derivation of a first order explicit finite difference scheme of the IBVP for two-point boundary condition is presented which is analogous to the well known Lax-Friedrichs scheme. The Lax-Friedrichs scheme for our model is not straight-forward to implement and one needs to employ a simultaneous physical constraint and stability condition. Therefore, a mathematical analysis is presented in order to establish the physical constraint and stability condition of the scheme. The finite difference scheme is implemented and the graphical presentation of numerical features of error estimation and rate of convergence is produced. Numerical simulation results verify some well understood qualitative behavior of traffic flow.
\end{abstract}

\section{Introduction}

The problem of computer simulation techniques of the traffic flow models has become an important area in the field of numerical solution methods. Many research groups are involved in dealing with the problem with different kinds of traffic models (like fluiddynamic models, kinetic models, microscopic models etc.) for several decades. E.g. In [1], the author shows that if the kinematics wave model of freeway traffic flow in its general form is approximated by a particular type of finite difference equation, the finite difference results converge to the kinematics wave solution despite the existence of shocks in the latter. Errors are shown to be approximately proportional to the mesh spacing with a coefficient of proportionality that depends on the wave speed, on its rate of change with density, and on the slope and curvature of the initial density profile. The asymptotic errors are smaller than those of Lax's first-order, centered difference method which is also convergent. In [2], the author develops a finite difference scheme for a previously reported non-equilibrium traffic flow model. This scheme is an extension of Godunov's scheme to systems. It utilizes the solutions of a series of Riemann problems at cell boundaries to construct approximate solutions of the non-equilibrium traffic flow model under general initial conditions. Moreover, the Riemann solutions at both left (upstream) and right (downstream) boundaries of a highway allow the specification of correct boundary conditions using state variables (e.g., density and/or speed) rather than 
fluxes. Preliminary numerical results indicate that the finite difference scheme correctly computes entropy-satisfying weak solutions of the original model.

In [3], the authors consider a mathematical model for fluid dynamic flows on networks which is based on conservation laws. The approximation of scalar conservation laws along arcs is carried out by using conservative methods, such as the classical Godunov scheme and the more recent discrete velocities kinetic schemes with the use of suitable boundary conditions at junctions. Riemann problems are solved by means of a simulation algorithm which processes each junction.

The above discussion gives us the motivation to study and investigate efficient finite difference scheme for the traffic flow simulation. With this ends, in this article, in section 2, we consider a fluid-dynamic traffic flow model which has been developed first by Lighthill and Whitham (1955) and Richard (1956) shortly called LWR model and present the basic features of the model based on Habermann (1977) in [4], Klar in [5]. The model describes traffic phenomena resulting from interaction of many vehicles by discussing the fundamental traffic variables like density, velocity and flow. In particular, a linear velocity-density closure relation yields a quadratic flux-density relation leads to formulate a first order non- linear partial differential equation. The exact solution of the non-linear PDE as a Cauchy problem is presented. However, in order to incorporate initial and boundary data, the non-linear first order partial differential is appended by initial and boundary value and formulates an initial boundary value problem (IBVP). Certainly, a numerical method is needed for the numerical implementation of the IBVP in practical situation and it is completely unavoidable to use numerical method to solve real traffic flow problem.

Therefore, based on the study of general finite difference method for first order non-linear PDE from Leveque 1992 [6], we present a first order explicit finite difference scheme, which is analogous to the well-known Lax-Friedrich scheme, for our considered traffic flow model as an IBVP with two-sided boundary condition. The scheme is not straight forward to implement and one needs to employ a simultaneous physical constraint and stability condition. Thus, in section 4 , we perform mathematical analysis and establish the physical constraints and stability condition. We develop necessary computer programming code for the implementation of the numerical scheme and perform numerical simulations in order to verify some qualitative traffic flow behavior for various traffic parameters. In section 5, we present the numerical features of error estimation and rate of convergence. We also present numerical simulations results in order to verify some well-understood qualitative traffic flow behavior.

\section{A Traffic Model based on a linear velocity-density function}

The fluid-dynamic traffic flow model is used to study traffic flow by collective variables such as traffic flow rate (flux) $q(x, t)$, traffic speed $v(x, t)$ and traffic density $\rho(x, t)$, all of which are functions of space, $x \in R$ and time, $t \in R^{+}$. The well-known LWR model ([4], [5], [7]) based on the principle of mass conservation reads as 


$$
\frac{\partial \rho}{\partial t}+\frac{\partial q}{\partial x}=0
$$

Inserting a linear velocity-density closure relationship

$$
v(\rho)=v_{\max }\left(1-\frac{\rho}{\rho_{\max }}\right)
$$

the flux $q$ takes the form

$$
q(\rho)=\rho v(\rho)=\rho \cdot v_{\max }\left(1-\frac{\rho}{\rho_{\max }}\right)
$$

and (1) leads to formulate a nonlinear first order hyperbolic partial differential equation (PDE) of the form

$$
\frac{\partial \rho}{\partial t}+\frac{\partial}{\partial x}\left(v_{\max }\left(\rho-\frac{\rho^{2}}{\rho_{\max }}\right)\right)=0
$$

The graph of the non-linear flux function given by equation (3) is known as Fundamental Diagram as sketched below.

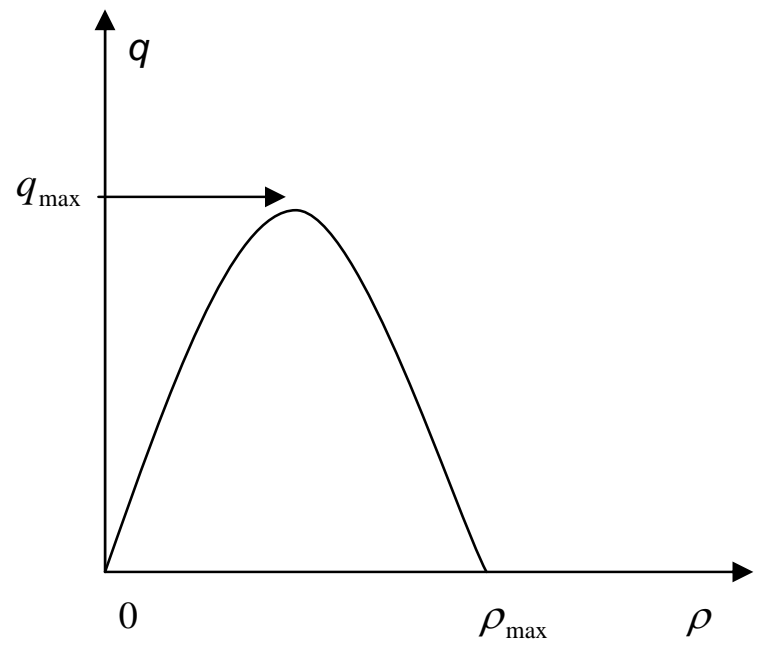

Figure 1: Fundamental Diagram of Traffic Flow

\section{Exact solution of the non-linear PDE by the method of characteristics}

The nonlinear PDE (4) can be solved if we know the traffic density at a given initial time, i.e., if we know the traffic density at a given initial time $t_{0}$ we can predict the traffic density for all future time $t \geq t_{0}$, in principle. Then we have to solve an initial value problem (IVP) of the form 


$$
\left\{\begin{array}{l}
\frac{\partial \rho}{\partial t}+\frac{\partial}{\partial x}\left(v_{\max }\left(\rho-\frac{\rho^{2}}{\rho_{\max }}\right)\right)=0 \\
\rho\left(t_{0}, x\right)=\rho_{0}(x)
\end{array}\right.
$$

The exact solution of the IVP (5) is given by

$$
\rho(t, x)=\rho\left(0, x_{0}\right)=\rho_{0}\left(x_{0}\right)=\rho_{0}\left(x-v_{\max }\left(1-\frac{2 \rho}{\rho_{\max }}\right) t\right)
$$

However, in reality it is very complicated to approximate the initial density $\rho_{0}(x)$ of the Cauchy problem as a function of $t$ from given initial data. Therefore, there is a demand of some efficient numerical methods for solving the IVP (5). Nevertheless, considering a simple form of initial value, the analytic solution (6) can be used to perform an error estimation of the numerical scheme.

\section{Lax-Friedrichs scheme for the numerical solution of the IBVP}

In this section we present the derivation of the finite difference scheme for our model with linear density-velocity relation appended with initial and two point boundary condition. The first order scheme is analogous to the well-known Lax-Friedrichs scheme [6]. We establish the physical constraints and stability condition for our version of LaxFriedrichs scheme. For this, we consider the traffic model (4) as an IBVP with two-point boundaries as below.

$$
\left(\text { IBVP) } \left\{\begin{array}{ll}
\frac{\partial \rho}{\partial t}+\frac{\partial q(\rho)}{\partial x}=0, t_{0} \leq t \leq T, a \leq x \leq b \\
\text { with I.C. } \quad \rho\left(t_{0}, x\right)=\rho_{0}(x) ; a \leq x \leq b \\
\text { and B.C. } \quad \rho(\mathrm{t}, \mathrm{a})=\rho_{\mathrm{a}}(t) ; t_{0} \leq t \leq T \\
& \rho(\mathrm{t}, \mathrm{b})=\rho_{\mathrm{b}}(t) ; t_{0} \leq t \leq T
\end{array}\right.\right.
$$

where, by equation (3), $q(\rho)=\rho \cdot v_{\max }\left(1-\frac{\rho}{\rho_{\max }}\right)$.

As we consider that the cars are running only in the positive $x$-direction, so the speed must be positive, i.e. in the range of $\rho, \quad q^{\prime}(\rho)=v_{\max }\left(1-\frac{2 \rho}{\rho_{\max }}\right) \geq 0$

Discretizing the time derivative $\frac{\partial \rho}{\partial t}$ in the IBVP (7) at any discrete point $\left(t_{n}, x_{i}\right)$ for $i=1, \cdots, M ; n=0, \cdots, N-1$; by the forward difference formula 


$$
\frac{\partial \rho\left(t_{n}, x_{i}\right)}{\partial t} \approx \frac{\rho_{i}^{n+1}-\rho_{i}^{n}}{\Delta t}
$$

and the spatial derivative $\frac{\partial q}{\partial x}$ by the central difference formula

$$
\frac{\partial q\left(t_{n}, x_{i}\right)}{\partial x} \approx \frac{q_{i+1}^{n}-q_{i-1}^{n}}{2 \Delta x}
$$

Inserting (9) and (10) in (7), the discrete version of the non-linear PDE formulates the first order finite difference scheme of the form

$$
\rho_{i}^{n+1}=\rho_{i}^{n}-\frac{\Delta t}{2 \Delta x}\left[q\left(\rho_{i+1}^{n}\right)-q\left(\rho_{i-1}^{n}\right)\right], \quad i=1, \cdots, M ; n=0, \cdots, N-1
$$

where $q\left(\rho_{i}^{n}\right)=v_{\max }\left(\rho_{i}^{n}-\frac{\left(\rho_{i}^{n}\right)^{2}}{\rho_{\max }}\right)$

In the finite difference scheme, the initial data $\rho_{i}^{0}$ for all $i=1, \cdots, M$; is the discrete versions of the given initial value $\rho_{0}(x)$ and the boundary data $\rho_{a}^{n}$ and $\rho_{b}^{n}$ for all $n=0, \cdots, N-1$ are the discrete versions of the given boundary values $\rho_{a}(x)$ and $\rho_{b}(x)$ respectively.

Unfortunately, despite the quite natural derivation of the scheme (11), it suffers from severe stability problems and is useless in practice. But if we replaces $\rho_{i}^{n}$ by $\frac{1}{2}\left(\rho_{i-1}^{n}+\rho_{i+1}^{n}\right)$ then the unstable scheme (11) becomes stable provided $\frac{\Delta t}{\Delta x}$ is sufficiently small, as it will be proved in the next section's proposition. Then (11) takes the form

$$
\rho_{i}^{n+1}=\frac{1}{2}\left(\rho_{i-1}^{n}+\rho_{i+1}^{n}\right)-\frac{\Delta t}{2 \Delta x}\left[q\left(\rho_{i+1}^{n}\right)-q\left(\rho_{i-1}^{n}\right)\right] ; i=1, \cdots, M ; n=0, \cdots, N-1
$$

This difference equation is known as Lax-Friedrichs scheme. Now we study the stability condition of this scheme for our model in which,

$$
q\left(\rho_{i}^{n}\right)=v_{\max }\left(\rho_{i}^{n}-\frac{\left(\rho_{i}^{n}\right)^{2}}{\rho_{\max }}\right) .
$$

\section{Stability and Physical Constraints conditions}

The implementation of Lax-Friedrichs scheme is not straight forward. Since the car is moving in one direction, so the characteristic speed $\frac{d q}{d t}$ must be positive. 


$$
\begin{aligned}
& \text { i.e. } \quad q^{\prime}\left(\rho_{i}^{n}\right)=v_{\max }\left(1-\frac{2 \rho_{i}^{n}}{\rho_{\max }}\right) \geq 0 \\
& \Rightarrow \rho_{\max } \geq 2 \rho_{i}^{n} \\
& \therefore q^{\prime}\left(\rho_{i}^{n}\right) \leq v_{\max }
\end{aligned}
$$

Proposition: The stability and physical constraint condition of the Lax-Friedrichs scheme (13) is guaranteed by the simultaneous conditions respectively

$$
\frac{v_{\max } \Delta t}{\Delta x} \leq 1 \text { and } \rho_{\max }=k \max _{i} \rho_{0}\left(x_{i}\right), k \geq 2 .
$$

Proof: Rewriting the non-linear PDE in (7) as $\frac{\partial \rho}{\partial t}+q^{\prime}(\rho) \frac{\partial \rho}{\partial x}=0$, the Lax-Friedrichs scheme (13) takes the form

$$
\begin{aligned}
\rho_{i}^{n+1} & =\frac{1}{2}\left(\rho_{i-1}^{n}+\rho_{i+1}^{n}\right)-\frac{1}{2} q^{\prime}\left(\rho_{i}^{n}\right) \frac{\Delta t}{\Delta x}\left[\rho_{i+1}^{n}-\rho_{i-1}^{n}\right] \\
& =\frac{1}{2}\left(\rho_{i-1}^{n}+\rho_{i+1}^{n}-\lambda \rho_{i-!}^{n}+\lambda \rho_{i+1}^{n}\right)
\end{aligned}
$$

where $\lambda:=q^{\prime}\left(\rho_{i}^{n}\right) \frac{\Delta t}{\Delta x}$

$$
\Rightarrow \rho_{i}^{n+1}=\frac{1}{2}\left[(1+\lambda) \rho_{i-1}^{n}+(1-\lambda) \rho_{i+1}^{n}\right]
$$

which is weighted arithmetic mean or simply weighted mean of $\rho_{i-1}^{n}$ and $\rho_{i+1}^{n}$ for $\lambda \leq 1$.

The equation (16) implies that if $\lambda \leq 1$, the new solution is a convex combination of the two previous solutions. That the solution at new time-step $(n+1)$ at a spatial-node $i$, is an average of the solutions at the previous time-step at the spatial-nodes $i-1$ and $i+1$. This means that the extreme value of the new solution is the average of the extreme values of the previous two solutions at the two consecutive nodes. Therefore, the new solution continuously dependent of the initial value $\rho_{i}^{0}, i=1, \cdots, M$ and the LaxFriedrichs scheme is stable for

$$
\lambda:=q^{\prime}\left(\rho_{i}^{n}\right) \frac{\Delta t}{\Delta x} \leq 1
$$

Then by the condition (15), the stability condition (18) can be guaranteed by

$$
\gamma:=\frac{v_{\max } \Delta t}{\Delta x} \leq 1
$$


Thus whenever one employs the stability condition (19), the physical constraints condition (14) can be guaranteed immediately by choosing

$$
\rho_{\max }=k \max _{i} \rho_{0}\left(x_{i}\right), k \geq 2 \text {. }
$$

\section{Numerical results and discussion}

We implement the Lax-Friedrichs scheme by developing a computer programming code and perform numerical simulation as described below.

\section{Error Estimation of the Numerical Scheme}

In order to perform error estimation, we consider the exact solution (6) with initial condition $\rho_{0}(x)=\frac{1}{2} x$, we have $\rho(t, x)=\rho_{0}\left(x_{0}\right)=\frac{1}{2}\left(x-v_{\max }\left(1-\frac{2 \rho}{\rho_{\max }}\right) t\right)$

$$
\Rightarrow \rho(t, x)=\frac{\left(x-v_{\max } t\right) / 2}{1-t v_{\max } / \rho_{\max }}
$$

We prescribe the corresponding two-sided boundary values by the equations

and

$$
\begin{aligned}
& \rho_{a}(t)=\rho\left(t, x_{a}\right)=\frac{x_{a}-v_{\max } t / 2}{1-v_{\max } t / \rho_{\max }} \\
& \rho_{b}(t)=\rho\left(t, x_{b}\right)=\frac{x_{b}-v_{\max } t / 2}{1-v_{\max } t / \rho_{\max }}
\end{aligned}
$$

For the above initial and boundary conditions with $v_{\max }=0.167(0.1 \mathrm{~km} / \mathrm{sec})=60.12$ $\mathrm{km} /$ hour, satisfying the physical constraint condition (20) $\rho_{\max }=5 \max _{i} \rho_{0}\left(x_{i}\right)=250 / \mathrm{km}$ in the spatial domain [5 km, $10 \mathrm{~km}$ ], we perform the numerical experiment for 4 minutes in $\Delta t=0.1$ time steps for a highway of $5 \mathrm{~km}$ in 101 spatial grid points with step size $\Delta x=100$ meters $=0.25$ which guarantees the stability condition (19) $\gamma=0.0668<1$. We compute the relative error in $L_{1}$-norm defined by:

$$
\|e\|_{1}:=\frac{\left\|\rho_{e}-\rho_{h}\right\|_{1}}{\left\|\rho_{e}\right\|_{1}}
$$

for all time where $\rho_{e}$ is the exact solution (6) and $\rho_{h}$ is the numerical solution computed by the Lax-Friedrichs scheme.

Figure 2 shows the relative errors for Lax-Friedrichs scheme, the relative errors remain below 0.00004 which is quite acceptable. It is obseved that the error is decreasing with respect to the smaller descretization parameters $\Delta t$ and $\Delta x$, which shows a very good feature of convergence of the Lax- Friedrichs scheme. 


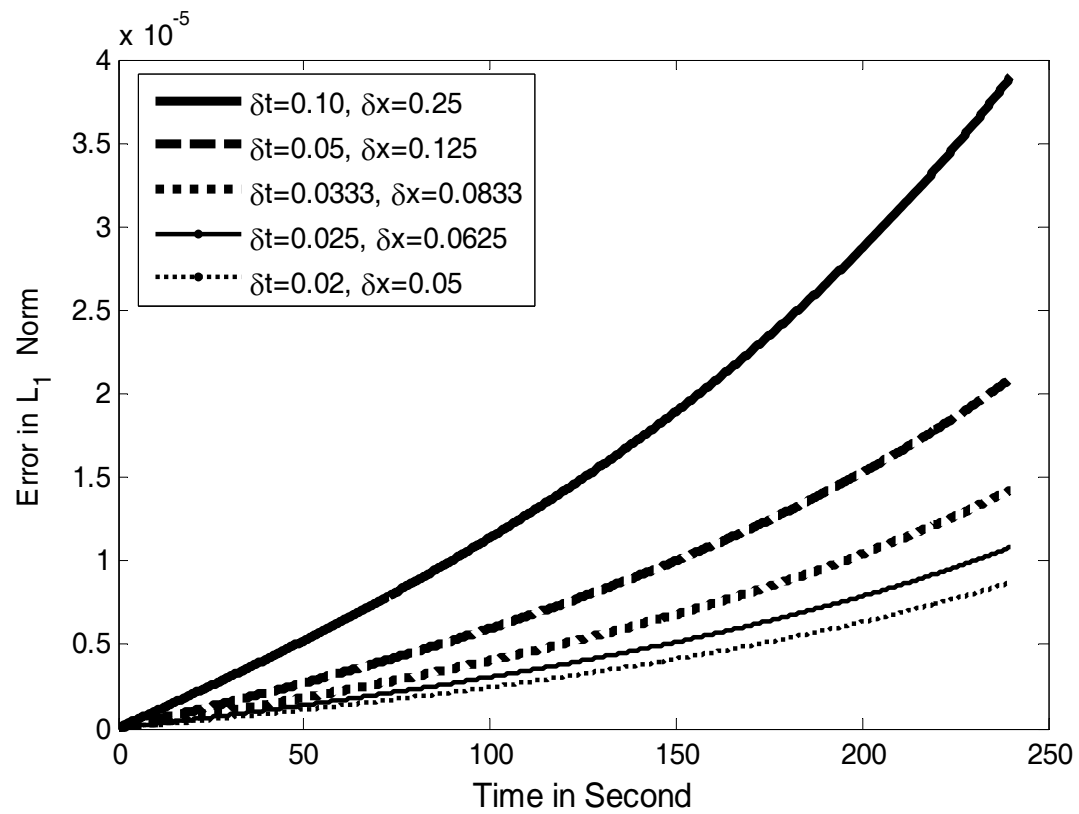

Figure 2: Convergence of Lax-Friedrichs Scheme

Now we consider the initial density using sine function and perform numerical computation in the spatial domain $[0,10]$ in $\mathrm{km}$. Figure 3(a) shows the initial density and the density after six minutes. 3(b) shows the propagating traffic waves at two min, four min and six min for the Lax-Friedrichs Scheme.

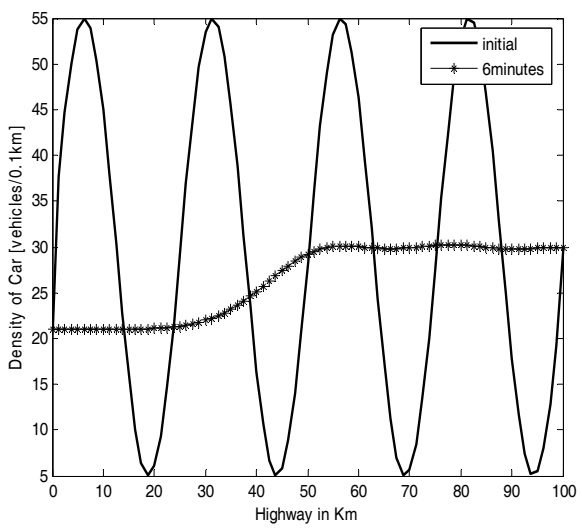




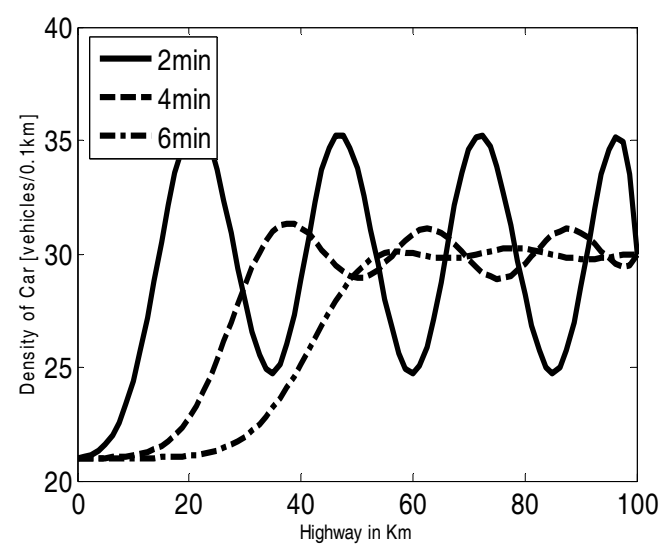

Figure 3(a): Initial Density Profile
Figure 3(b): Time evolution of density profile

Figure 4(a) and 4(b) shows the density $u(t, x)$ profiles and velocity $v(t, x)$ profiles for three different times and one can read from the two figures that the density and velocity are maintaining the negative relation, as given by equation (2), throughout the computational process as expected.

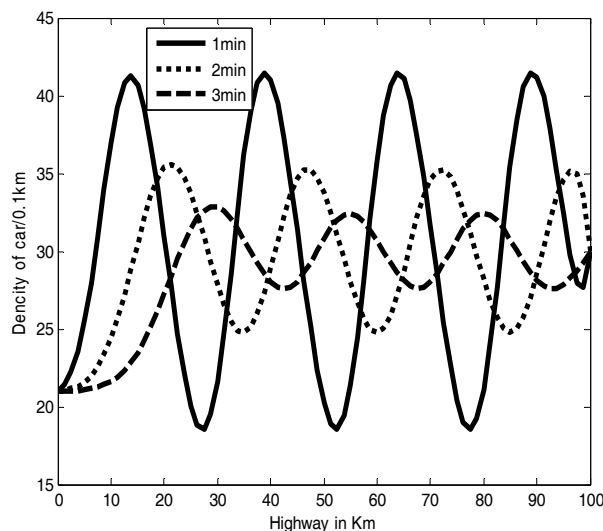

Figure 4(a): Density profiles

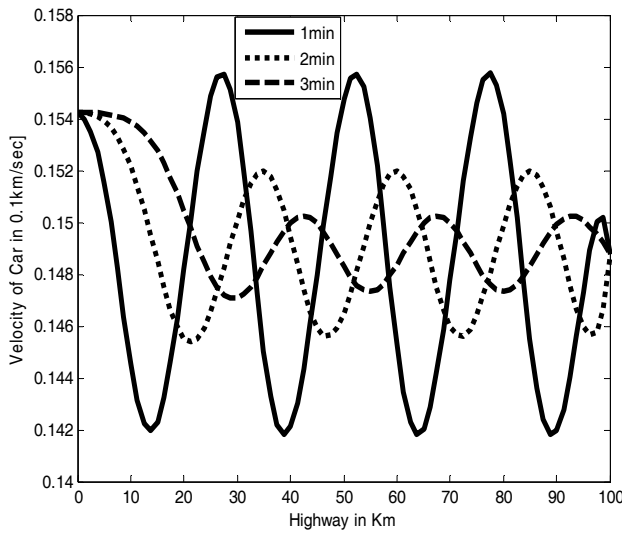

Figure 4(b): Velocity profiles 


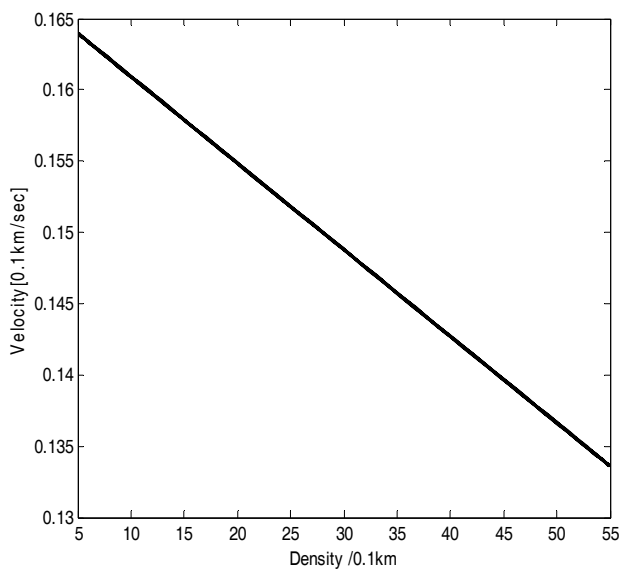

Figure 5(a): Traffic velocity as a function of density

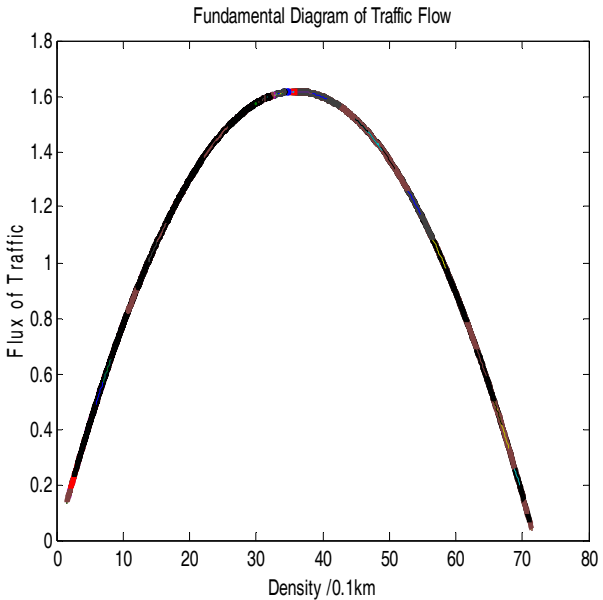

Figure 5(b): Traffic Flux as a function of density

Finally, Figure 5(a) presents the computed car velocity as a function of density and Figure 5(b) shows the computed flux (traffic flow) as a function of density. Figure 5(b) verifies qualitative behavior, the well-known Fundamental diagram as Figure 1.

\section{Conclusion}

From the numerical results we observed that our version of Lax-Friedrichs scheme for the considered traffic flow model is adequate for traffic flow simulation. The time-step in the established stability condition and physical constraints condition is not stiff and this resulted computational efficiency of the scheme. The computational results showed the accuracy up to five decimal places and a good rate of convergence. The numerical simulation results verified some well-known qualitative traffic flow behavior. The scheme can be extended for multi-lane traffic flow simulations which we left for future work.

\section{REFERENCES}

1. Carlos F. Daganzo. "A finite difference approximation of the kinematic wave model of traffic flow”, Transportation Research Part B: Methodological Volume 29, Issue 4, (Elsevier), p.261- 276,1995 .

2. H. M. Zhang. “A finite difference approximation of a non-equilibrium traffic flow model”, Transportation Research Part B: Methodological Volume 35, Issue 4, (Elsevier), p. 337-365, 2001.

3. Gabriella Bretti, Roberto Natalini, Benedetto Piccoli, “A Fluid-Dynamic Traffic Model on Road Networks”, Comput Methods Eng., (C) CIMNE, Barcelona, Spain 2007.

4. Richard Haberman, "Mathematical models”, Prentice-Hall, Inc., 1977.

5. Axel Klar, Reinhart D. Kuhne and Raimurd Wegener, “Mathematical models for Vehicular Traffic”, Preprint, Dept. of Math., TU of Kaiserlautern, Germany, 1996.

6. Randall J. LeVeque, “Numerical Methods for Conservation Laws”, second Edition, 1992, Springer.

7. Clive L. Dym, “Principles of Mathematical Modeling”, Academic press, 2004.

8. L S Andallah, Shajib Ali, M O Gani, M K Pandit \& J Akhter “A Finite Difference Scheme for a Traffic Flow Model based on a Linear Velocity-Density Function” JU J. of Sci. Vol 32, No. 1, 2009. 
